Agr. Biol. Chem., 38 (3), 641 644, 1974

\title{
Polyacrylamide Gel Electrophoresis of Plasteins
}

\author{
Shun-Jen Tsai, Michiko Yamashita, Soichi Arai \\ and Masao Fujmaki \\ Department of Agricultural Chemistry, University of Tokyo, Tokyo \\ Received October 25, 1973
}

\begin{abstract}
A peptic hydrolysate of soybean protein was filtered with Sephadex G-25 and was separated approximately into four fractions (I, II, II, and IV in the order of mol. wt.). Fraction II (av. mol. wt.: 1043) and III (av. mol. wt.: 685) were more plastein-productive than others. When plastein produced from Fraction II with Nagarse was investigated by plate electrophoresis using $7.5 \%$ polyacrylamide-gel, the upper limit of the molecular weight was found to be about 25,000. A similar result was obtained also with Fraction III. The increase of molecular weight in the course of the plastein formation with the mixture (substrate) of Fractions II and III was shown that the final product lay mainly in a position between cytochrome $c$ (mol. wt.: 11,700) and Nagarse (mol. wt.: 27,600). In addition, the gel-electrophoretic experiments revealed that the most favorable condition for the plastein synthesis were $\mathrm{pH} 6.5$ and $35 \%$ in substrate concentration.
\end{abstract}

The molecular weight of plastein has been estimated by the trichloroacetic acid (TCA) precipitation, ${ }^{1 \sim 3)}$ gel filtration, ${ }^{2)}$ membrane transmittance ${ }^{3}$ and so on. Although Tauber" has reported that a plastein has a molecular weight of $250,000 \sim 500,000$ in an associated state, Yamashita et al. have presented a value of about 5500 in a case" and about 9690 in another case, ${ }^{5)}$ according to chemical determination. The present paper describes the plastein productivity and the molecular weight increase observed by polyacrylamide gel electrophoresis.

\section{MATERIALS AND METHODS}

Substrates. The peptic hydrolysate (degree of hydrolysis*: $85 \%$ ) of soybean globulin was prepared according to Yamashita et al. ${ }^{2}$ The hydrolysate was filtered with a Toyo-roshi filter paper, No. 2 to obtain a soluble fraction. This soluble fraction ( $1 \mathrm{~g}$ on a dry-matter basis) was subjected to gel filtration using Sephadex G-25 under the conditions described in the legend to Fig. 1, to obtain four fractions, Frs. I, II, III, and IV, with yields of $0.17,0.47,0.16$, and $0.09 \mathrm{~g}$, respectively. Chemical analysis ${ }^{5 j}$ showed that the average molecular weight of Fr. II was 1043 and that of Fr. III was 685; whereas Frs. I and IV were higherand lower-molecular than Fr. II and Fr. III, respectively. Because Frs. I and IV were not plastein-productive,

\footnotetext{
* $(10 \%$ TCA-soluble N/total N) 100.
}

Frs. II and III were, individually or in combination, used as substrates for the plastein synthesis.

Plastein synthesis. The reaction was performed as following unless otherwise noted; substrate was dissolved in water (final $\mathrm{pH}, 6.5$; final concentration, $35 \%$ and incubated with a $1 / 100$ amount of nagarse (subtilisin $\mathrm{BPN}^{\prime}$; Nagase Sangyo Co.) at $37^{\circ} \mathrm{C}$ for $24 \mathrm{hr}$, if not noted in particular.

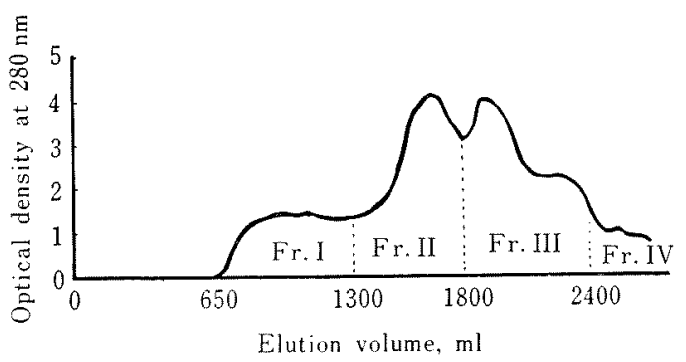

FIG. 1. Gel Filtration of the Soluble Hydrolysate of Soybean Protein.

Gel: Sephadex G-25. Gel bed volume: $4 \therefore 140$ $\mathrm{cm}$. Solvent: $0.01 \mathrm{~N} \mathrm{HCl}$. Flow rate: $120 \mathrm{ml} / \mathrm{hr}$ $\left(5^{\circ} \mathrm{C}\right)$.

Polyacrylamide gel electrophoresis. A $7.5 \%$ polyacrylamide gel plate was prepared as usual ${ }^{6}$ and equilibrated with phenol-acetic acid-water $(1: 1: 1)^{6,7}$ for $10 \mathrm{hr}$ before being submitted to electrophoresis. The electrophoresis of each sample applied to the plate was carried out at $3 \mathrm{~mA} / \mathrm{cm}^{2}$ for $7 \mathrm{hr}$ at room temperature. Thereafter, the zones in the plate were transcribed to 
a filter paper, and the paper was treated with $1 \%$ ninhydrin in $n$-butanol. The plate was then stained with Amido-black $10 \mathrm{~B}$ (Kanto Chemical Co.) to make plastein zones visible.

Calibration. Cytochrome c (mol. wt.: 11,700), trypsin (mol. wt.: 23,500), Nagarse (mol. wt.: 26,700), and ovalbumin (mol. wt.: ca. 45,000) were used as markers in the above electrophoresis and a calibration curve shown in Fig. 2 was obtained between the relative migration distances of the markers and their semilogarithumic molecular weights. The relative migration distances were obtained based on the migration distance of glycine (mol. wt.: 75).

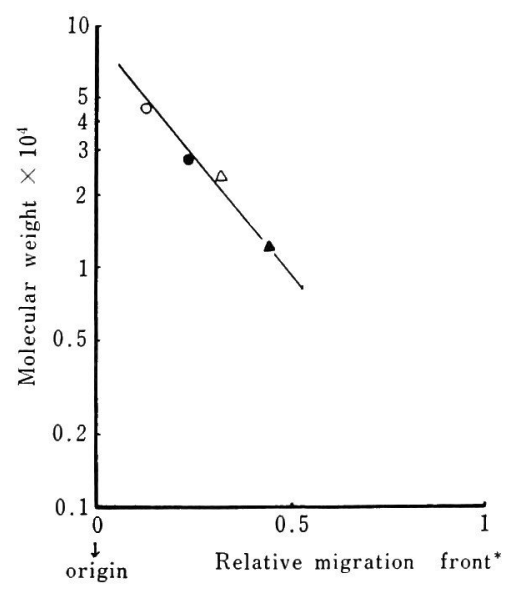

FIG. 2. A Relative Migration-Molecular Weight Calibration by $7.5 \%$ Polyacrylamide Gel Electrophoresis.

O, ovalbumin; - , Nagarse; $\Delta$, trypsin; $\Delta$, cytochrome $c$.

* Migration distance of glycine.

\section{RESULTS AND DISCUSSION}

It has been generally shown that the plastein reaction requires at least three special conditions; moleuclar size of substrate, ${ }^{2)}$ substrate concentration, ${ }^{8)}$ and $\mathrm{pH}^{9}{ }^{9)}$ As to the molecular size of substrate it has been demonstrated $^{2)}$ that the substrate is required to be moderately low-molecular weight material, whereas higher- or lower-moleculr substrate is not plastein-productive even if other two conditions are favorablly adjusted. The present study also showed that neither Fr. I (polypeptide fraction) nor Fr. IV (small peptide and free amino acid fraction) produced any significant
Table I. Plastein Productivities of THE Four Fractions

Percent amount of $10 \%$ TCA-insoluble substance after incubation with enzyme without enzyme

$\begin{array}{lrr}\text { Fr. I } & 6.6 & 3.3 \\ \text { Fr. II } & 23.0 & 0.0 \\ \text { Fr. III } & 33.2 & 0.0 \\ \text { Fr. IV } & 00 & 0.0\end{array}$

amount of plastein in practice (Table I). OI the contrary, the two fractions, Frs. II and III gave plasteins with yields of approximatel! 20 and $30 \%$, respectively (Table I).

Comparing the molecular size of Fr. II witl that of the product from this fraction, thi polyacrylamide gel electrophoresis gave a clear visible difference as shown in Fig. 3, in whicl the product was found to grow up to the siz

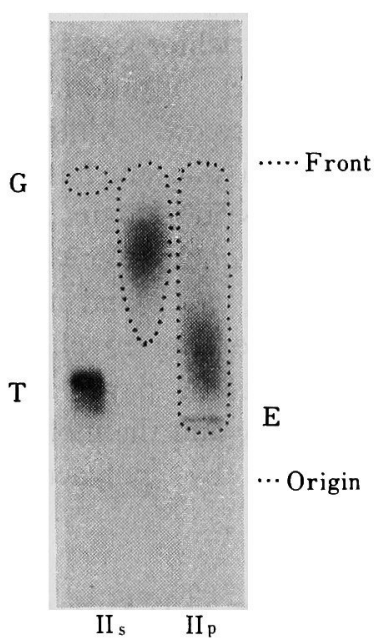

FIG. 3. Electrophoretic Patterns of a Plastein anc Its Substrate.

$\mathrm{II}_{\mathrm{p}}$ : plastein from Fr. II. $\mathrm{II}_{\mathrm{s}}$ : substrate (Fr. II) T: Trypsin (marker). G: Glycine. E: Enzym band (Nagarse). Photographed patterns: Amido black staining. Drawn patterns: Transcribed anc ninhydrin-treated.

of trypsin. A similar result was obtained als from Fr. III (Fig. 4); in this case before th plastein reaction Fr. III was hardly stained $b$ Amidoblack $10 \mathrm{~B}$, probably because of bein low-molecular.

Next, the examination of the molecule 
weight increase during the plastein reaction was carried out with a 3:1 mixture of Frs. II and III by electrophoresis. When this mixed substrate was subjected to the plastein reaction, a gradual increase in molecular weight (i.e.,

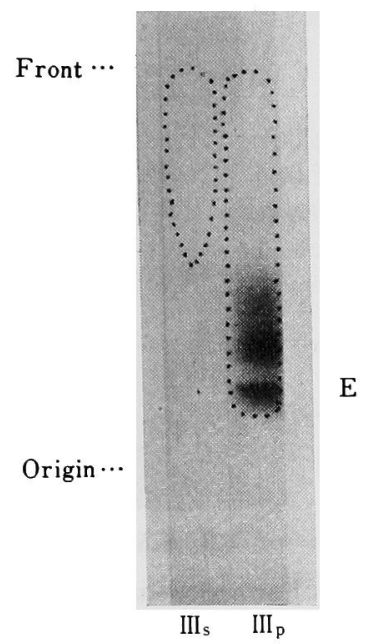

FIG. 4. Electrophoretic Patterns of a Plastein and Its Substrate.

III $_{\mathrm{p}}$ : Plastein from Fr. III. III I $_{\mathrm{s}}$ Substrate (Fr. III). E: Enzyme band (Nagarse). Photographed patterns: Amidoblack staining. Drawn patterns: Transcribed and ninhydrin-treated.

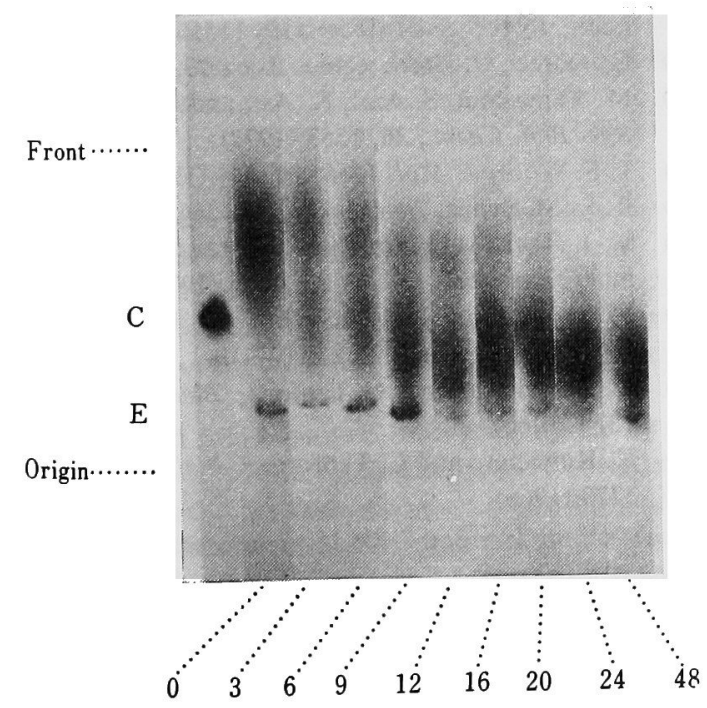

FIg. 5. Time-course Profile of Plastein Formation by Polyacrylamide Gel Electrophoresis.

Figures: Incubation times. C: Cytochrome $c$ (marker). E: Enzyme band (Nagarse). decrease in migration distance) was observed in the course of the reaction time (Fig. 5). Although the migration distance of the substrate at the beginning was longer than that of cytochrome $c$, the final product detected by the Amidoblack staining was found to locate mainly between cytochrome $c$ and Nagarse.

In order to confirm the average molecular weight of this plastein fraction, ultracentrifugal sedimentation analysis was carried out on this fraction. The analytical conditions were described in the legend to Fig. 6 . As a result, the sedimentation coefficient, $s_{20, \mathrm{w}}$, and the molecular weight were roughly estimated to be $1.04 \mathrm{~S}$ and 20,000 , respectively, by the use of parameters reported in an experiment under similar conditions. ${ }^{10,11)}$

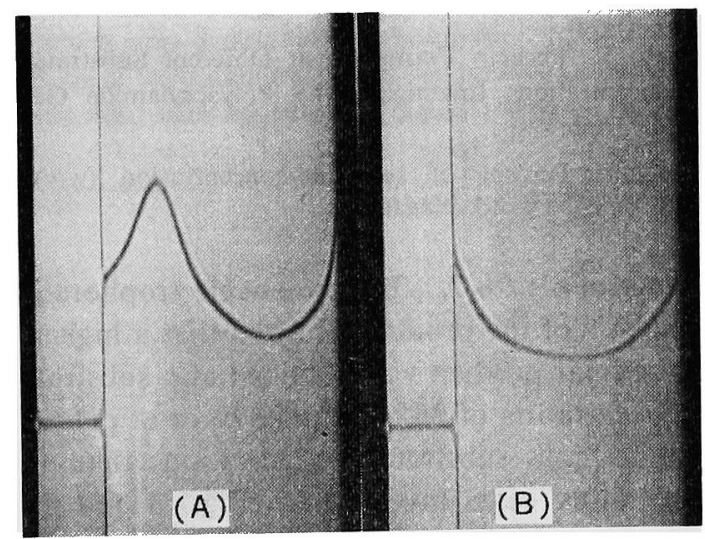

FIG. 6. Ultracentrifugal Sedimentation Patterns of Plastein (A) and Its Substrate (B) in Synthetic Boundary Cells.

Apparatus: Hitachi Ultracentrifugal Analyzer-1A. Solvent: $6 \mathrm{~m}$ guanidine hydrochloride.

Concentration: $0.5 \%$.

Revolution velocity: $60,000 \mathrm{rpm}$. Bar angle: $65^{\circ}$. Pictures taken at $30 \mathrm{~min}$ after having reached 60,000 rpm.

As mentioned before, the substrate concentration and the $\mathrm{pH}$ of the reaction mixture must be adjusted also properly in order to make the plastein formation reaction proceed effectively. As to the substrate concentration, Tsai et al. ${ }^{81}$ have demonstrated that the plastein formation is the most effective at $35 \%$. Concerning to the $\mathrm{pH}$ dependence of Nagarse, it has been found ${ }^{9}$ that the optimum lies in the 


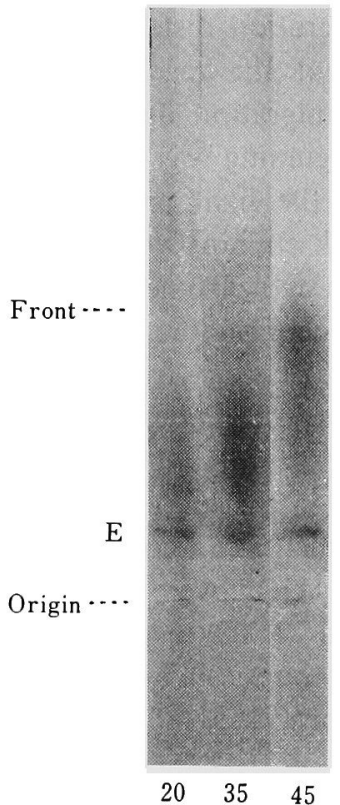

FIG. 7. Plastein Formation at Different Substrateconcentrations Investigated by Polyacrylamide Gel Electrophoresis.

Figures: Percent of substrate-concentration (w/v). E: Enzyme band (Nagarse).

range of $\mathrm{pH} 6 \sim 7$. The present electrophoretic analysis of the products showed that a highermolecular product was obtained at a substrate concentration of $35 \%$ (at $\mathrm{pH} 6.5$ ) or at $\mathrm{pH} 6.5$ (at $35 \%$ in substrate concentration) than in any other cases investigated (Figs. 7 and 8). These results have been confirmed in the previous work. ${ }^{8,9)}$

Acknowledgement. This research has been financed in part by grants made by National Food Research Institute, Ministry of Agriculture and Forestry, and by Ryoshoku Kenkyu Kai.

\section{REFERENCES}

1) M. Fujimaki, M. Yamashita, S. Arai and H.

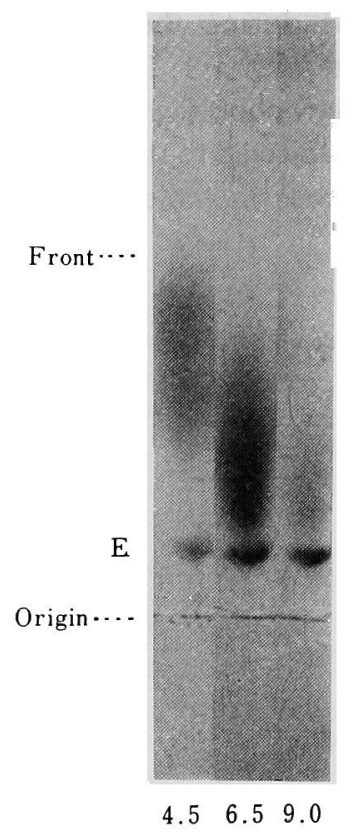

FIG. 8. Plastein Formation at Different $\mathrm{pH}$-conditions Investigated by Polyacrylamide Gel Electrophoresis.

Figures: pH-values of the system.

E: Enzyme band (Nagarse).

Kato, Agr. Biol. Chem., 34, 1325 (1970).

2) M. Yamashita, S. Arai, J. Matsuyama, M. Gonda, H. Kato and M. Fujimaki, ibid., 34, 1484 (1970).

3) M. Yamashita, S. Arai, S.-J. Tsai and M. Fujimaki, J. Agr. Food Chem., 19, 1151 (1971).

4) H. Tauber, J. Amer. Chem. Soc., 73, 1288 (1951).

5) M. Yamashita, S. Arai, K. Aso and M. Fujimaki, Agr. Biol. Chem., 36, 1353 (1972).

6) T. S. Work, J. Mol. Biol., 10, 544 (1964).

7) R. L. M. Synge, Biochem. J., 65, 266 (1957).

8) S. -J. Tsai, M. Yamashita, S. Arai and M. Fujimaki, Agr. Biol. Chem., 36, 1045 (1972).

9) M. Yamashita, S. -J. Tsai, S. Arai, H. Kato and M. Fujimaki, ibid., 35, 86 (1971).

10) K. Kawahara and C. Tanford, Biochemistry, 5, 1578 (1966).

11) K. Kawahara and C. Tanford, J. Biol. Chem., 241, 3228 (1966). 